\title{
Pilot-Based LMMSE Channel Estimation for OFDM Systems With Power-Delay Profile Approximation
}

\author{
Kun-Chien Hung and David W. Lin, Senior Member, IEEE
}

\begin{abstract}
In linear-minimum-mean-square-error (LMMSE) channel estimation for multicarrier systems, one needs to know the channel correlation function. This poses a problem for systems with a small number of pilots operating in time-varying channels. We propose to approximate the channel power-delay profile (PDP) with a shape that can completely be described in two parameters, namely, the mean delay and the root-mean-square (RMS) delay spread. Furthermore, we develop a simple technique to estimate these delay parameters. The approximate PDP is then used to generate the LMMSE filter coefficients for data-subcarrier channel estimation. Mathematical expressions are derived that can be used to predict the accuracy of the various estimates, and they are verified by simulation. The proposed technique is applicable to both point-to-point communication and multiaccess communication where different users may experience different channel conditions. As a practical application, we also specialize the proposed technique to Mobile WiMAX signals and investigate the resulting performance.
\end{abstract}

Index Terms-Channel estimation, IEEE 802.16e, Mobile WiMAX, orthogonal frequency-division multiple access (OFDMA), orthogonal frequency-division multiplexing (OFDM), rootmean-square (RMS) delay spread.

\section{INTRODUCTION}

$\mathbf{P}$ ILOT-AIDED channel estimation is widely employed in today's coherent wireless orthogonal frequency-division multiplexing (OFDM) systems. Often, the subcarriers that carry pilot signals are scattered in frequency and in time. One favored approach to OFDM channel estimation is linear-minimummean-square-error (LMMSE) estimation, i.e., Wiener filtering [1]-[4]. Given some initial channel estimates at pilot subcarriers, standard LMMSE optimization leads to the LMMSE channel estimate at any subcarrier $d$ as [3], [5]

$$
\hat{H}_{d}=\mathbf{w}_{d}^{H} \hat{\mathbf{h}}_{p}
$$

with

$$
\mathbf{w}_{d}=\left(\mathbf{R}_{p}+\sigma_{n}^{2} \mathbf{I}\right)^{-1} \mathbf{r}_{d p}
$$

Manuscript received October 6, 2008; revised March 31, 2009 and June 29, 2009. First published August 18, 2009; current version published January 20, 2010. This work was supported by the National Science Council of the Republic of China under Grant NSC 96-2219-E-009-003. The review of this paper was coordinated by Prof. Y. Ma.

K.-C. Hung was with the Department of Electronics Engineering, National Chiao Tung University, Hsinchu 30010, Taiwan. He is now with MediaTek Inc., Hsinchu 30078, Taiwan (e-mail: hkc.ee90g@nctu.edu.tw).

D. W. Lin is with the Department of Electronics Engineering, National Chiao Tung University, Hsinchu 30010, Taiwan (e-mail: dwlin@mail.nctu.edu.tw).

Color versions of one or more of the figures in this paper are available online at http://ieeexplore.iee.org.

Digital Object Identifier 10.1109/TVT.2009.2029862 where $\hat{H}_{d}$ is the desired channel estimate; $\mathbf{w}_{d}$ is the vector of Wiener filter coefficients; superscript $H$ denotes Hermitian transpose; $\hat{\mathbf{h}}_{p}$ is the vector of given initial channel estimates; $\mathbf{R}_{p}=E\left(\mathbf{h}_{p} \mathbf{h}_{p}^{H}\right)$ (with $E$ denoting expectation), where $\mathbf{h}_{p}$ is the vector of true channel responses at pilot subcarriers; $\mathbf{r}_{d p}=$ $E\left(\mathbf{h}_{p} H_{d}^{*}\right)$, where $H_{d}$ is the true channel response at subcarrier $d ; \sigma_{n}^{2}$ is the variance of additive noise in $\hat{\mathbf{h}}_{p}$ [assumed white Gaussian, i.e., additive white Gaussian noise (AWGN)]; and I denotes an identity matrix. A common method to estimate $\hat{\mathbf{h}}_{p}$ is the least-squares (LS) method, which merely divides the received signal value at each pilot subcarrier by the pilot value there to obtain the estimated response there [3]. Then, if the pilots have unity magnitude, $\sigma_{n}^{2}$ is equal to the variance of the additive channel noise.

To perform LMMSE estimation, one needs to know $\mathbf{R}_{p}, \mathbf{r}_{d p}$, and $\sigma_{n}^{2}$. The estimation of $\sigma_{n}^{2}$ is usually not a problem, as it can be done by measuring the received power at the (typically ample) null subcarriers. The estimation of $\mathbf{R}_{p}$ and $\mathbf{r}_{d p}$, however, poses a problem for two reasons. One is that accurate estimation of correlation values requires averaging over sufficiently many samples. However, when the channel is time varying, one may not have this luxury within the coherence time, particularly if the system only transmits a small number of pilots. The other reason concerns $\mathbf{r}_{d p}$, wherein each element is the crosscorrelation of the channel response at a pilot subcarrier and that at subcarrier $d$. Suppose the pilots are of the comb type, i.e., equally spaced in frequency, but the spacing is different from that between subcarrier $d$ and any pilot. Then, the estimation of $\mathbf{r}_{d p}$ would itself require interpolation (which involves approximation) in addition to averaging. As a result, strictsense LMMSE channel estimation may be impractical in many situations.

To sidestep the aforementioned problem, one approach is to employ a simple model for the channel power-delay profile (PDP). Two common choices are the exponential model [3], [6], [7] and the uniform model [7]-[9]. For these PDPs, the entire second-order channel statistics are defined by the mean delay $\tau_{\mu}$ and the root-mean-square (RMS) delay spread $\tau_{\text {rms }}$. Given $\tau_{\mu}$ and $\tau_{\text {rms }}$, one can calculate $\mathbf{R}_{p}$ and $\mathbf{r}_{d p}$ and then the Wiener filter $\mathbf{w}_{d}$ for any $\sigma_{n}^{2}$. The price paid, however, is that the PDP model employed may be an oversimplification of the actual situation, resulting in a modeling error that needs to be reckoned with when considering the overall channel-estimation performance. This point will later be given further attention.

Several estimation methods for the RMS delay spread have been proposed. Witrisal et al. [10], [11] show that this delay spread is proportional to the level-crossing rate of the channel 
frequency response. However, accurate estimation of the levelcrossing rate requires dense frequency sampling of the channel response, whereas many OFDM systems employ widely spaced pilots. Athaudage and Jayalath [12] exploit a relation between the cyclic-prefix (CP) correlation and the RMS delay spread of the exponential PDP. However, the method may require the use of many OFDM symbols. It also heavily draws on the exponential PDP condition. Yücek and Arslan [13] propose several methods based on some frequency-domain correlation functions of the channel response or the received signal. The amount of computation is relatively high.

In this paper, we derive a simple method to estimate the mean delay and the RMS delay spread from noisy channel response samples. As the purpose of delay estimation is to facilitate LMMSE channel estimation, the underlying considerations are somewhat different from when delay estimation itself is the goal. This issue is addressed in Section II. Then, Section III derives the estimation method for the channel delay parameters, and Section IV examines its accuracy. Subsequently, Section V considers LMMSE channel estimation based on the estimated delay parameters. As a practical application, Section VI specializes the proposed technique to Mobile WiMAX signals [14], [15]. Finally, Section VII concludes the paper.

\section{Channel Modeling for Channel Estimation}

Consider a discrete-time equivalent low-pass channel impulse response

$$
h(n)=\sum_{l=0}^{L-1} \alpha_{l} \delta(n-l)
$$

where $n$ and $l$ are integers in units of the sampling period $T_{s}$, and $\alpha_{l}$ is the complex gain of path $l$. The mean delay and the RMS delay spread are given by, respectively

$$
\begin{aligned}
\tau_{\mu} & =\frac{\sum_{l=0}^{L-1} E\left(\left|\alpha_{l}\right|^{2}\right) l}{\sum_{l=0}^{L-1} E\left(\left|\alpha_{l}\right|^{2}\right)} \\
\tau_{\mathrm{rms}} & =\sqrt{\frac{\sum_{l=0}^{L-1} E\left(\left|\alpha_{l}\right|^{2}\right)\left(l-\tau_{\mu}\right)^{2}}{\sum_{l=0}^{L-1} E\left(\left|\alpha_{l}\right|^{2}\right)} .}
\end{aligned}
$$

While the last two equations describe familiar concepts, the meaning of $E\left(\left|\alpha_{l}\right|^{2}\right)$ therein should be interpreted according to application. For example, if the delay parameters are used in system design for a certain terrain, then the expectation should be taken over channel conditions pertaining to that terrain. For our present channel estimation problem, suppose that one estimation is performed for $K$ OFDM symbols. Then, the expectation (or better, the average) should be taken over these symbols. In the extreme case of $K=1, n o$ average should be taken, but the instantaneous channel response in that symbol period should be used to compute $\tau_{\mu}$ and $\tau_{\text {rms }}$.

For complexity and performance reasons, in practical designs, channel estimation is often performed after carrier frequency and OFDM symbol timing are synchronized. However, experience shows that typical symbol timing synchronizers may yield some error in identifying the initial path delay. Moreover, in multiaccess communication, variation in the transmission timing of different users will cause some user channels to have nonzero initial delays even under perfect symbol timing synchronization. Hence, we assume that the channel estimator input contains no carrier frequency error, but the PDP may have a possibly nonzero initial delay $\tau_{0}$. (Conventional PDPs are obtained with initial channel delays excised.)

Fourier transforming the PDP gives the frequency autocorrelation function of the channel. For an exponential PDP, we have

$$
R_{f}(k) / R_{f}(0)=\frac{e^{-j 2 \pi \tau_{0} k / N}}{1+j 2 \pi \tau_{\mathrm{rms}} k / N}
$$

where $R_{f}(k)$ denotes the value of the autocorrelation function at lag $k, \tau_{0}=\tau_{\mu}-\tau_{\mathrm{rms}}$, and $N$ is the discrete-Fouriertransform (DFT) size used in multicarrier signaling. For a uniform PDP of width $T$

$$
R_{f}(k) / R_{f}(0)=\frac{e^{-j 2 \pi \tau_{\mu} k / N} \sin (\pi T k / N)}{\pi T k / N}
$$

where $\tau_{\mu}=\tau_{0}+T / 2$, and $T=\sqrt{12} \tau_{\text {rms. }}$.

\section{Estimation of Channel Delay Parameters}

The frequency response of the channel (3) is given by

$$
H(f)=\sum_{l=0}^{L-1} \alpha_{l} e^{-j 2 \pi l f / N}
$$

where the division by $N$ in the exponent normalizes the period of $H(f)$ in $f$ to $N$. If we advance the channel response by $\tau$ (arbitrary) time units, then the frequency response becomes

$$
H_{a}(f)=e^{j 2 \pi \tau f / N} H(f)=\sum_{l=0}^{L-1} \alpha_{l} e^{-j 2 \pi(l-\tau) f / N} .
$$

Differentiating $H_{a}(f)$ with respect to $f$, we get

$$
\frac{d H_{a}(f)}{d f}=\frac{-j 2 \pi}{N} \sum_{l=0}^{L-1} \alpha_{l}(l-\tau) e^{-j 2 \pi(l-\tau) f / N} .
$$

By Parseval's theorem

$$
\left\langle\left|\frac{d H_{a}(f)}{d f}\right|^{2}\right\rangle=\frac{4 \pi^{2}}{N^{2}} \sum_{l=0}^{L-1}\left|\alpha_{l}\right|^{2}(l-\tau)^{2}
$$

where $\langle\cdot\rangle$ denotes frequency averaging. Taking expectation

$$
E\left\langle\left|\frac{d H_{a}(f)}{d f}\right|^{2}\right\rangle=\frac{4 \pi^{2}}{N^{2}} \sum_{l=0}^{L-1} E\left(\left|\alpha_{l}\right|^{2}\right)(l-\tau)^{2} \triangleq \bar{J}(\tau) .
$$

The aforementioned provides an alternative characterization of the mean delay and the RMS delay spread. First, it is easily shown that $\bar{J}(\tau)$ is minimized when $\tau=\tau_{\mu}$. Second

$$
\tau_{\mathrm{rms}}^{2}=\frac{N^{2} \min \bar{J}(\tau)}{4 \pi^{2} \sum_{l=0}^{L-1} E\left(\left|\alpha_{l}\right|^{2}\right)} .
$$


This characterization leads to a novel way of estimating $\tau_{\mu}$ and $\tau_{\text {rms }}$ that is particularly amenable to OFDM transmission. To see how, consider a system where one out of every $F_{s}$ subcarriers is a pilot. (The formulation can be extended to deal with unequally spaced pilots, but the equations become somewhat cumbersome.) Approximate $d H_{a}(f) / d f$ by first-order difference, e.g., $\left[H_{a}\left(\left(f+F_{s}\right) \% N\right)-H_{a}(f)\right] / F_{s}$, where " $\%$ " denotes modulo operation, and substitute it into the first equality in (12). (The modulo operation arises due to the cyclic nature of the frequency spectrum of a discretetime signal [16].) Then, after some straightforward algebra, we obtain

$$
\bar{J}(\tau) \approx \frac{1}{F_{s}^{2}} E\left\langle\left|e^{j \phi} H\left(\left(f+F_{s}\right) \% N\right)-H(f)\right|^{2}\right\rangle_{p}
$$

where $\phi=2 \pi \tau F_{s} / N, f$ takes values only over pilot frequencies, and $\langle\cdot\rangle_{p}$ denotes averaging over pilot subcarriers, and we have assumed that $\left(f+F_{s}\right) \% N$ is a pilot subcarrier. Now, let $R_{i}$ be the (instantaneous) frequency-domain autocorrelation of the channel response

$$
R_{i}=\left\langle H\left(\left(f+i F_{s}\right) \% N\right) H^{*}(f)\right\rangle_{p} .
$$

Then, from (14), we have

$$
\bar{J}(\tau) \approx \frac{2}{F_{s}^{2}}\left[E\left(R_{0}\right)-\Re\left\{e^{j \phi} E\left(R_{1}\right)\right\}\right] .
$$

In (14), the operation inside the absolute value delimiters can be interpreted as filtering of $H(f)$ in the frequency domain by a filter with impulse response $e^{j \phi} \delta(f)-\delta\left(f-F_{s}\right)$. By the modulation theorem of Fourier analysis [16], it amounts to multiplying the channel impulse response $h(n)$ by the inverse DFT of $e^{j \phi} \delta(f)-\delta\left(f-F_{s}\right)$, i.e., $e^{j \phi}-e^{j 2 \pi F_{s} n / N}(0 \leq n \leq$ $N-1)$. By Parseval's theorem, therefore, frequency averaging of $\left|e^{j \phi} H\left(\left(f+F_{s}\right) \% N\right)-H(f)\right|^{2}$ yields the same result as time averaging of $\left|h(n)\left(e^{j \phi}-e^{j 2 \pi F_{s} n / N}\right)\right|^{2}$. Since $e^{x} \approx 1+x$ for small $x$, if $L$ is small enough that $2 \pi F_{s}(L-1) / N \ll 1$, then $e^{j \phi}-e^{j 2 \pi F_{s} l / N} \approx-j 2 \pi F_{s}(l-\tau) / N$, for $0 \leq l \leq L-1$. Moreover, by sampling theory, when $L-1<N / F_{s}$ (as implied by $\left.2 \pi F_{s}(L-1) / N \ll 1\right)$, the subsampled averaging (over pilots only) in (14) causes no time-aliasing error and yields the same result as averaging over all frequencies. Then, (16) gives an accurate approximation of $\bar{J}(\tau)$, defined in (12). In any case, (16) gives an approximation of $\bar{J}(\tau)$, regardless of the condition.

The derivation leading to (16) indicates that $\tau_{\mu}$ and $\tau_{\text {rms }}$ can be estimated in the following way: 1) Estimate the channel responses at the pilot subcarriers; 2) estimate $R_{i}(i=0,1)$; 3) estimate $\bar{J}(\tau) ; 4)$ find the value of $\tau$ that minimizes $\bar{J}(\tau)$ (which yields an estimate of $\tau_{\mu}$ ); and 5) substitute the result into (13) (which yields an estimate of $\tau_{\text {rms }}^{2}$ ). Some details follow.

Step 1 can be achieved using the LS method. Let $\hat{H}(f)$ denote the resulting channel estimate at pilot subcarrier $f$, which is assumed to deviate from the true $H(f)$ by AWGN of variance $\sigma_{n}^{2}$. As mentioned, we may estimate $\sigma_{n}^{2}$ from the received power in the null subcarriers of the system. Let $\hat{\sigma}_{n}^{2}$ denote the resulting estimate. Then, in step $2, R_{0}$ and $R_{1}$ can be estimated via

$$
\hat{R}_{0}=\left\langle|\hat{H}(f)|^{2}\right\rangle_{p}-\hat{\sigma}_{n}^{2}, \quad \hat{R}_{1}=\left\langle\hat{H}\left(\left(f+F_{s}\right) \% N\right) \hat{H}^{*}(f)\right\rangle_{p} .
$$

Thus, for step $3, \bar{J}(\tau)$ can be estimated using

$$
\hat{J}_{\mathrm{Av}}(\tau) \triangleq \frac{2}{F_{s}^{2}}\left[\operatorname{Av}\left(\hat{R}_{0}\right)-\Re\left\{e^{j \phi} \operatorname{Av}\left(\hat{R}_{1}\right)\right\}\right]
$$

where "Av" denotes time averaging over the OFDM symbols for which channel estimation is performed. (The number of such symbols has been denoted by $K$.) For step 4, we may estimate the mean delay as

$$
\hat{\tau}_{\mu} \triangleq \arg \min _{\tau} \hat{J}_{\mathrm{Av}}(\tau)=-\frac{N \angle \operatorname{Av}\left(\hat{R}_{1}\right)}{2 \pi F_{s}}
$$

which also yields $\min \hat{J}_{\mathrm{Av}}(\tau)=2\left[\operatorname{Av}\left(\hat{R}_{0}\right)-\left|\operatorname{Av}\left(\hat{R}_{1}\right)\right|\right] / F_{s}^{2}$. Finally, for step 5, in view of (13) and that $R_{0}=\left\langle|H(f)|^{2}\right\rangle_{p} \approx$ $\sum_{l=1}^{L}\left|\alpha_{l}\right|^{2}$, we may estimate $\tau_{\text {rms }}$ as

$$
\hat{\tau}_{\mathrm{rms}}=\frac{N}{2 \pi F_{s}} \sqrt{2\left[1-\frac{\left|\operatorname{Av}\left(\hat{R}_{1}\right)\right|}{\operatorname{Av}\left(\hat{R}_{0}\right)}\right]} .
$$

\section{Estimation Accuracy of the Delay Parameters}

The accuracy of $\hat{\tau}_{\mu}$ and $\hat{\tau}_{\text {rms }}$ is analyzed in the Appendix. In summary, (25) and (28) give the mean of $\Delta \tau_{\mu} \triangleq \hat{\tau}_{\mu}-\tau_{\mu}$, and (37) gives the variance. Furthermore, (39) and (42) give the mean of $\Delta \tau_{\mathrm{rms}}^{2} \triangleq \hat{\tau}_{\mathrm{rms}}^{2}-\tau_{\mathrm{rms}}^{2}$, and (48) gives its the variance. Moreover, both $\Delta \tau_{\mu}$ and $\Delta \tau_{\text {rms }}^{2}$ are Gaussian in high SNRs. We evaluate the estimation accuracy by simulation below.

We simulate an OFDM system with bandwidth $=10 \mathrm{MHz}$, DFT size $=1024$, and CP length $=128$. The channel has six Rayleigh paths with power profile $[0,-1,-9,-10,-15,-20]$ (in decibels) and delay profile $[10,13,17,21,27,35]$ (in samples), which follow the International Telecommunications Union's Vehicular A channel model [17], except that the path delays are rounded to integer sample numbers and an initial delay of ten samples (approximately $0.9 \mu \mathrm{s}$ ) is added. We consider estimating the instantaneous delay parameters, i.e., $K=1$. This is useful, for example, in channel estimation based on only one received OFDM symbol. By using the least amount of data possible, the estimation is most prone to noise effects, as can also be seen in (37) and (48), where the leading factors are both inversely proportional to $K$. We consider SNRs between 0 and $40 \mathrm{~dB}$ and let the channel be block static (i.e., have a constant response in one OFDM symbol duration).

Consider first the mean errors in $\hat{\tau}_{\mu}$ and $\hat{\tau}_{\text {rms }}$. We generate a set of 100 channel realizations at random according to the aforementioned PDP. For each channel realization, we run 100 simulations (i.e., 100 OFDM symbols) for each simulated SNR. Fig. 1 shows the distributions of the mean values of $\Delta \tau_{\mu}$ and $\Delta \tau_{\text {rms }}^{2}$ over some 100 channel realizations at $\mathrm{SNR}=20 \mathrm{~dB}$, with pilot spacing $F_{s}=8$. We see that the mean errors are 

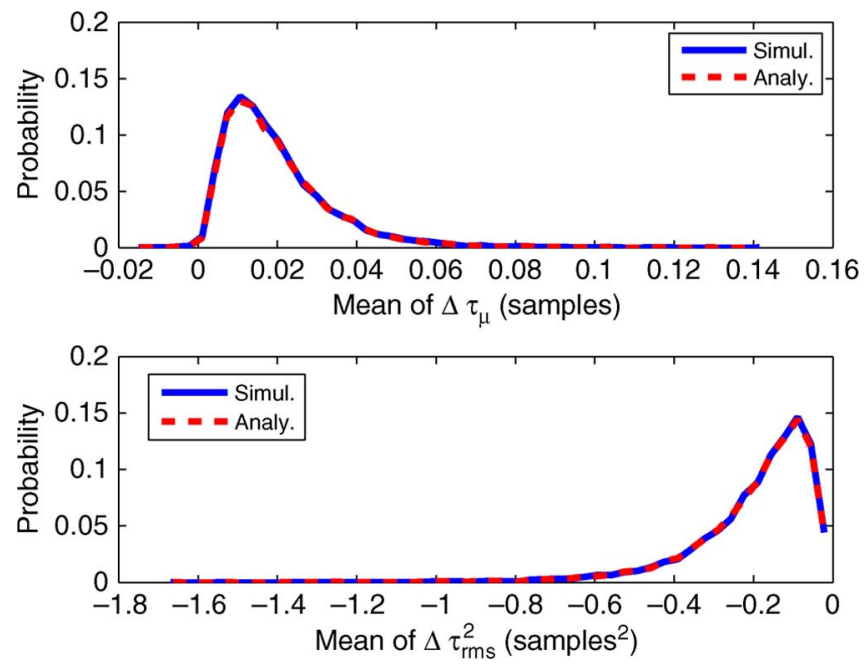

Fig. 1. Distribution of mean errors in delay estimation at $\mathrm{SNR}=20 \mathrm{~dB}$ over 100 random channel realizations. (Top) Mean of $\Delta \tau_{\mu}$. (Bottom) Mean of $\Delta \tau_{\mathrm{rms}}^{2}$.

quite small in all cases. Furthermore, there is a very close match between the distributions of analysis and simulation results.

Next, consider the standard deviations of $\Delta \tau_{\mu}$ and $\Delta \tau_{\mathrm{rms}}^{2}$. Fig. 2 shows their averaged values (over 100 random channel realizations and 100 OFDM symbols per channel realization) versus SNR under two pilot spacings: 8 and 16 . The theory and the simulation results agree well, except for very low SNRs. We also see that the pilot spacing does affect the estimation accuracy. Moreover, for $\Delta \tau_{\mu}$, the curves show a -1 slope at lower SNRs and a -0.5 slope at higher SNRs. This agrees with the presence of a $\sigma_{n}^{2}$ term in the brackets in (37), whose effect is more prominent in lower SNRs but diminishes in higher SNRs. For $\Delta \tau_{\text {rms }}^{2}$, the $\sigma_{n}^{2}$ terms in (48) indicate a similar slope transition with increasing SNRs, which is also clearly exhibited in Fig. 2.

We now consider how the channel estimator derived from $\hat{\tau}_{\mu}$ and $\hat{\tau}_{\text {rms }}$ performs.

\section{Channel Estimation Based on the Approximate POWER-DELAY PROFILE}

Consider the following channel-estimation procedure: 1) Perform LS estimation of the pilot subcarrier responses; 2) estimate the delay parameters according to (19) and (20); 3 ) obtain the autocorrelation function associated with the approximate PDP (where (6) and (7) correspond to exponential and uniform approximations, respectively); and 4) based on this autocorrelation function, do LMMSE filtering according to (1) and (2) to estimate the data-subcarrier responses.

\section{A. Complexity Analysis}

In the complexity analysis, we only examine steps 2 and 3, for therein lies the uniqueness of the proposed method, whereas steps 1 and 4 are rather common among LMMSE methods.

Let there be $P$ pilot subcarriers in an OFDM symbol. Then, step 2 needs some amount of computation to obtain $\hat{\sigma}_{n}^{2}$, on the order of $2 P$ complex multiply-and-adds per OFDM symbol to calculate $\hat{R}_{0}$ and $\hat{R}_{1}$, and then a few arithmetic operations per
$K$ OFDM symbols to obtain $\hat{\tau}_{\mu}$ and $\hat{\tau}_{\text {rms }}$. For step 3 , if the channel estimators have order $M$ and each data subcarrier response is estimated using the nearest $M$ pilot subcarrier responses, then the maximum correlation lag in (2) does not exceed $M F_{s}$. Thus, the complexity, per $K$ OFDM symbols, is no greater than that needed to compute $R_{f}(k)$ according to (6) or (7), for $|k| \leq M F_{s}$. The aforementioned should favorably compare with other methods of estimating the channel autocorrelation function, such as direct averaging of sample autocorrelations. The variety of possible conditions makes impossible a simple one-equation complexity comparison. Table I provides a comparison for a typical situation.

\section{B. Performance Analysis}

Now, consider the mean square error (MSE) of LMMSE channel estimation using an approximate PDP. For any linear estimator $\hat{\mathbf{w}}_{d}$ for subcarrier $d$, straightforward algebra shows that the MSE is given by

$$
\begin{aligned}
\sigma_{\hat{H}}^{2} & \triangleq E\left|\hat{\mathbf{w}}_{d}^{H} \hat{\mathbf{h}}_{p}-H_{d}\right|^{2} \\
& =E\left|H_{d}\right|^{2}-2 \Re\left\{\hat{\mathbf{w}}_{d}^{H} \mathbf{r}_{d p}\right\}+\hat{\mathbf{w}}_{d}^{H}\left(\mathbf{R}_{p}+\sigma_{n}^{2} \mathbf{I}\right) \hat{\mathbf{w}}_{d} .
\end{aligned}
$$

Let $\Delta \mathbf{w}_{d}=\hat{\mathbf{w}}_{d}-\mathbf{w}_{d}$, where $\mathbf{w}_{d}$ is the ideal LMMSE channel estimator obtained with true channel correlation values. Then

$$
\begin{aligned}
\sigma_{\hat{H}}^{2}= & E\left|H_{d}\right|^{2}-2 \Re\left\{\left(\mathbf{w}_{d}+\Delta \mathbf{w}_{d}\right)^{H} \mathbf{r}_{d p}\right\} \\
& +\left(\mathbf{w}_{d}+\Delta \mathbf{w}_{d}\right)^{H}\left(\mathbf{R}_{p}+\sigma_{n}^{2} \mathbf{I}\right)\left(\mathbf{w}_{d}+\Delta \mathbf{w}_{d}\right) \\
= & \underbrace{E\left|H_{d}\right|^{2}-\mathbf{r}_{d p}^{H}\left(\mathbf{R}_{p}+\sigma_{n}^{2} \mathbf{I}\right)^{-1} \mathbf{r}_{d p}}_{\triangleq \sigma_{H}^{2}} \\
& +\underbrace{\Delta \mathbf{w}_{d}^{H}\left(\mathbf{R}_{p}+\sigma_{n}^{2} \mathbf{I}\right) \Delta \mathbf{w}_{d}}_{\triangleq \Delta \sigma_{H}^{2}}
\end{aligned}
$$

where $\sigma_{H}^{2}$ is the MSE associated with ideal LMMSE channel estimation, and $\Delta \sigma_{H}^{2}$ is the excess MSE from using a nonideal channel estimator.

Equation (22) can be used to evaluate the MSE under either ideal or approximate LMMSE channel estimation. In the case of the proposed estimator, we have

$$
\hat{\mathbf{w}}_{d}=\left(\hat{\mathbf{R}}_{p}+\hat{\sigma}_{n}^{2} \mathbf{I}\right)^{-1} \hat{\mathbf{r}}_{d p}
$$

where $\hat{\mathbf{R}}_{p}$ and $\hat{\mathbf{r}}_{d p}$ are approximations of $\mathbf{R}_{p}$ and $\mathbf{r}_{d p}$, respectively, as obtained using the estimated delay parameters. A more specific characterization of $\sigma_{\hat{H}}^{2}$ beyond (22) appears difficult to obtain.

\section{Simulation With Comb-Type Pilots}

For the numerical assessment of the estimation performance, consider a system with the same parameters as in Section IV, except that pilot spacing $=4$. In addition to the Vehicular A channel, we also simulate the SUI-5 channel [18] with power profile $[0,-5,-10]$ (in decibels) and delay profile $[0,45,112]$ (in samples). The channels are subject to block-static fading. The channel estimator for each data subcarrier contains four taps. The channel estimation is based on only one OFDM 

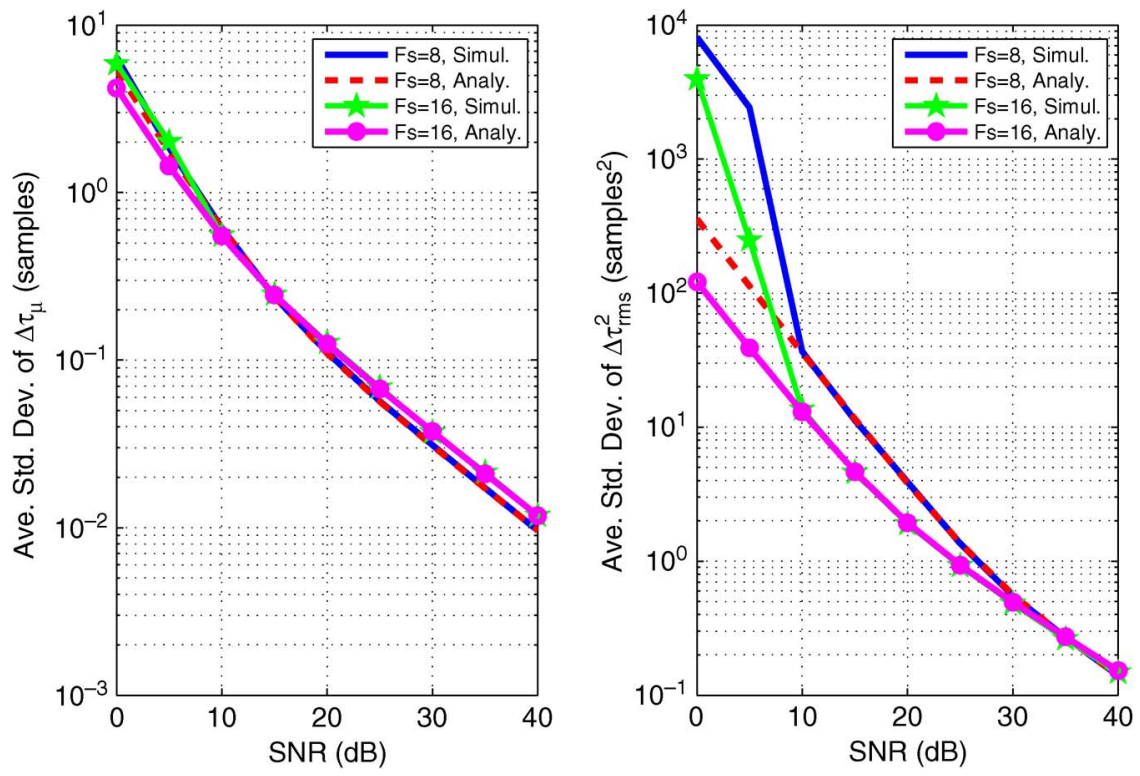

Fig. 2. Averaged standard deviation in delay estimation over 100 random channel realizations. (Left) Standard deviation in $\Delta \tau_{\mu}$. (Right) Standard deviation in $\Delta \tau_{\mathrm{rms}}^{2}$.

TABLE I

Complexity of DifFerent Techniques of AutocorRelation-FunCtion Estimation

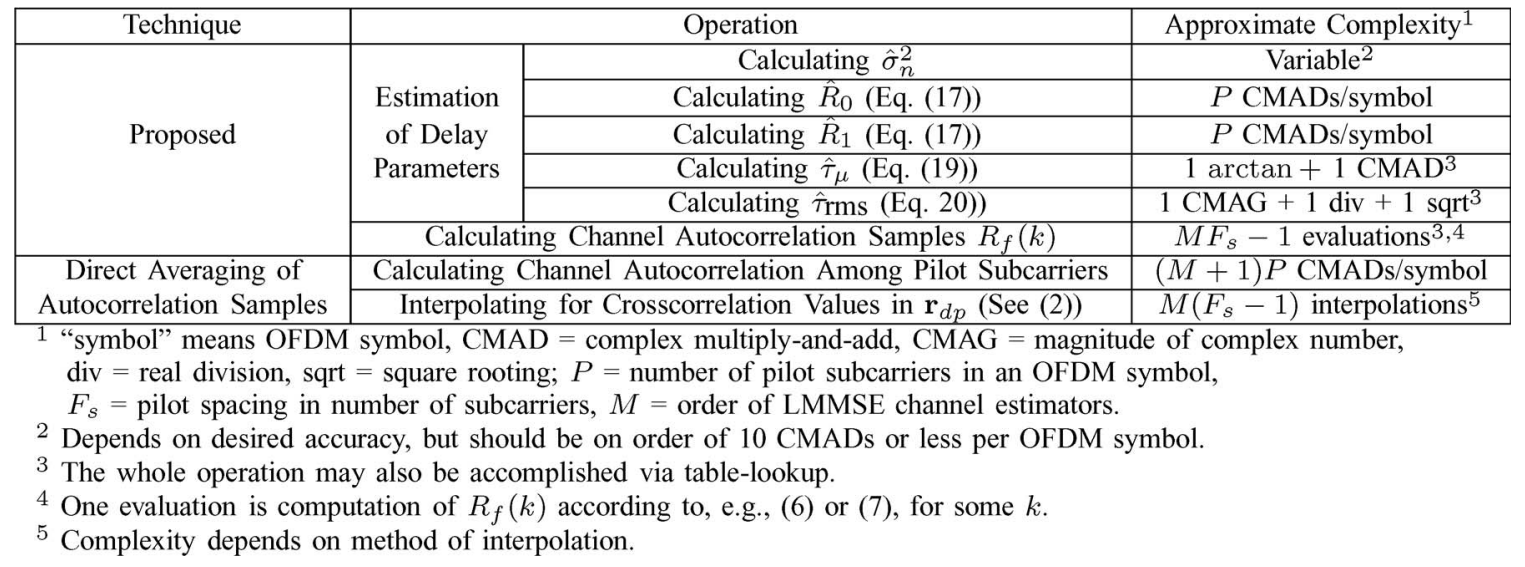

symbol, i.e., $K=1$; thus, $\tau_{\mu}$ and $\tau_{\text {rms }}$ are also estimated with $K=1$. For benchmarking purposes, ideal Wiener filtering based on true channel autocorrelation is also conducted.

Fig. 3 shows the channel estimation performance under various conditions in terms of the normalized MSE (NMSE) with respect to the channel power gain. The analytical result in each case is calculated using the true channel correlation values. Therefore, the deviation of simulation data from the analytical includes the effect of estimation errors in delay parameters. As the simulation and the analytical results closely match under all conditions, the estimation errors in the delay parameters have little effect.

In Vehicular A, the proposed technique performs quite close to ideal Wiener estimation, particularly when employing the exponential PDP model. In SUI-5, which has a much greater delay spread than Vehicular A, the proposed technique shows clear error floors in higher SNRs due to model errors. As model errors are unavoidable with PDP approximation, unless one changes the channel estimator length, all one can do is to choose a suitable PDP shape based on knowledge about the channel. In the case of SUI-5, that the exponential model performs much better than the uniform is intuitively unsurprising, in view of the shape of the true PDP.

\section{Application to Mobile WiMAX ChANNEL ESTIMATION}

The Mobile WiMAX system provides an interesting practical example to test the proposed channel estimation technique. In the downlink (DL), it divides the subcarriers in each OFDM symbol into "clusters," each consisting of 14 consecutive subcarriers in frequency. Each DL user's signal is contained in a set of clusters that are pseudorandomly distributed in frequency. The uplink (UL) transmission employs orthogonal frequency-division multiple access (OFDMA). Each UL user's signal comprises a set of "tiles," where each tile spans four consecutive subcarriers in frequency. The tiles pertaining to a user's signal are also pseudorandomly distributed in frequency. The structures of clusters and tiles vary with time and are illustrated in Fig. 4. The aforementioned pseudorandom 

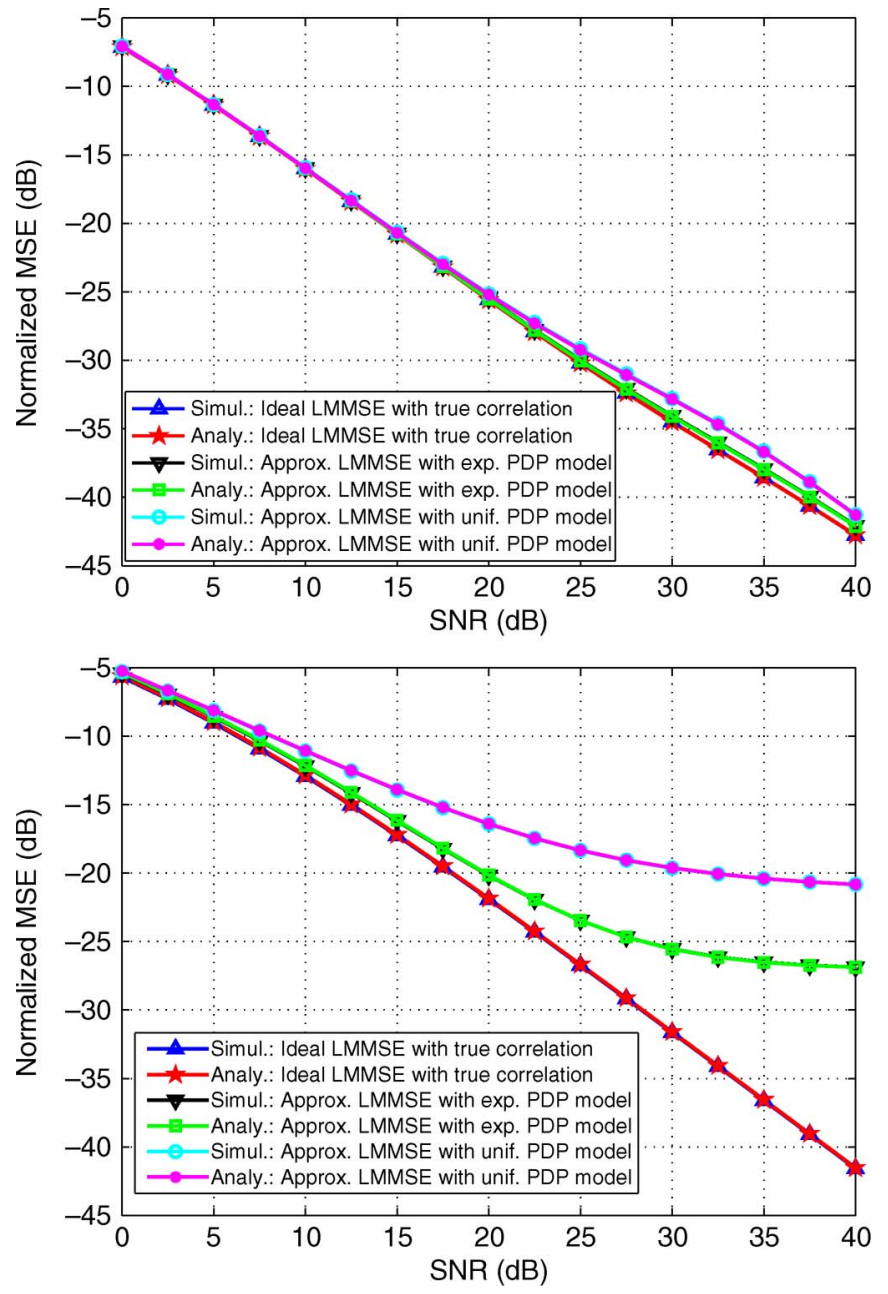

Fig. 3. NMSE in channel estimation for OFDM transmission with comb-type pilots. (Top) Vehicular A channel. (Bottom) SUI-5.

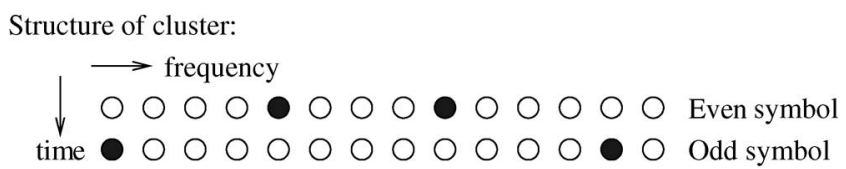

Structure of tile:

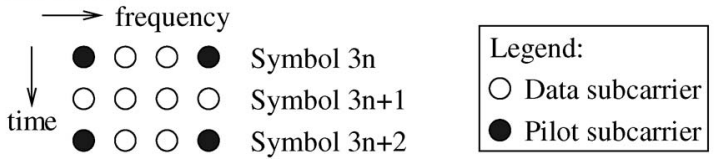

Fig. 4. Structures of cluster and tile.

distribution of subcarrier groups is incompatible with the underlying signal structure assumed in many studies on OFDM channel estimation.

\section{A. DL Channel Estimation}

By the pseudorandom frequency distribution, two closest clusters may not be next to each other in frequency but may be widely apart to the point well beyond the channel coherence bandwidth. Thus, it may not pay to perform LMMSE channel estimation across cluster boundaries. Hence, we separately do LMMSE filtering in individual clusters. Although there are only two pilots per cluster in any OFDM symbol, there are four

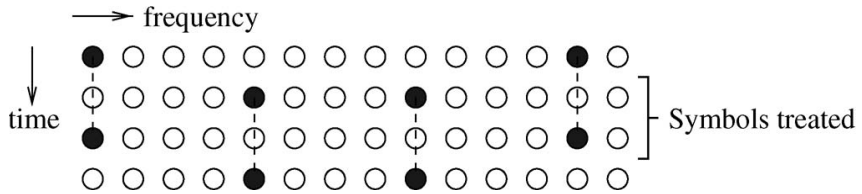

Fig. 5. Temporal interpolation of channel estimates at nonpilot subcarriers, assuming the middle two symbols are being considered for channel estimation. Dashed lines indicate where linear interpolations are performed.

frequencies where a pilot may appear over time. Hence, we try to have four-tap filters. Present-day OFDM systems usually operate under the condition where the product of peak Doppler shift $\left(f_{d}\right)$ and OFDM symbol time is on the order of 0.1 or less. Under this condition, channel variation over several consecutive OFDM symbols can be well approximated as linear [19], [20]. Therefore, we may do linear interpolation in time to produce two additional channel estimates in each cluster at the pilot positions of temporally adjacent OFDM symbols, as illustrated in Fig. 5.

We consider two ways of DL channel estimation. In one, the channel delay parameters for a user are reestimated once every pair of OFDM symbols. In the other, they are estimated only once per time-division-duplex (TDD) frame using the preamble symbol that begins DL subframe [14]. The first method has higher complexity, and the second should yield worse performance for time-varying channels. The first method proceeds as follows.

1) Estimate the noise variance using the null subcarriers in the preamble symbol.

2) Do LS channel estimation at the pilot subcarriers of all used clusters.

3) Linearly interpolate in time to acquire two additional channel estimates per cluster, as illustrated in Fig. 5.

4) Estimate the mean delay and the RMS delay spread using the channel estimates at all pilot subcarriers of all used clusters and obtain the four-tap Wiener channel estimators for the data subcarriers. (There are a total of ten such Wiener filters.)

5) Perform Wiener filtering to obtain the desired channel estimates.

The second channel estimation method is quite similar, except that the equivalent of step 4 is performed only once per DL subframe based on the received preamble symbol.

We simulate a system where FFT size $=1024$, bandwidth $=$ $10 \mathrm{MHz}$, TDD frame length $=5 \mathrm{~ms}$, and DL subframe size $=$ 1 preamble +24 OFDM symbols. We again consider the Vehicular A (with 10 samples of initial delay) and SUI-5 channels. The channels are again subject to block-static fading but at a rate corresponding to a carrier frequency of $2.5 \mathrm{GHz}$ and a mobile speed of $100 \mathrm{~km} / \mathrm{h}$.

Fig. 6 shows the NMSE results. In addition to the proposed methods, the performance of ideal LMMSE estimation with true channel correlation is also plotted for comparison. For the Vehicular A channel, both proposed methods perform close to the ideal LMMSE benchmark under either PDP model, particularly at lower SNR values. In SUI-5, which has a much larger delay spread than Vehicular A, however, the proposed methods start to show error floors in higher SNRs, whereas 

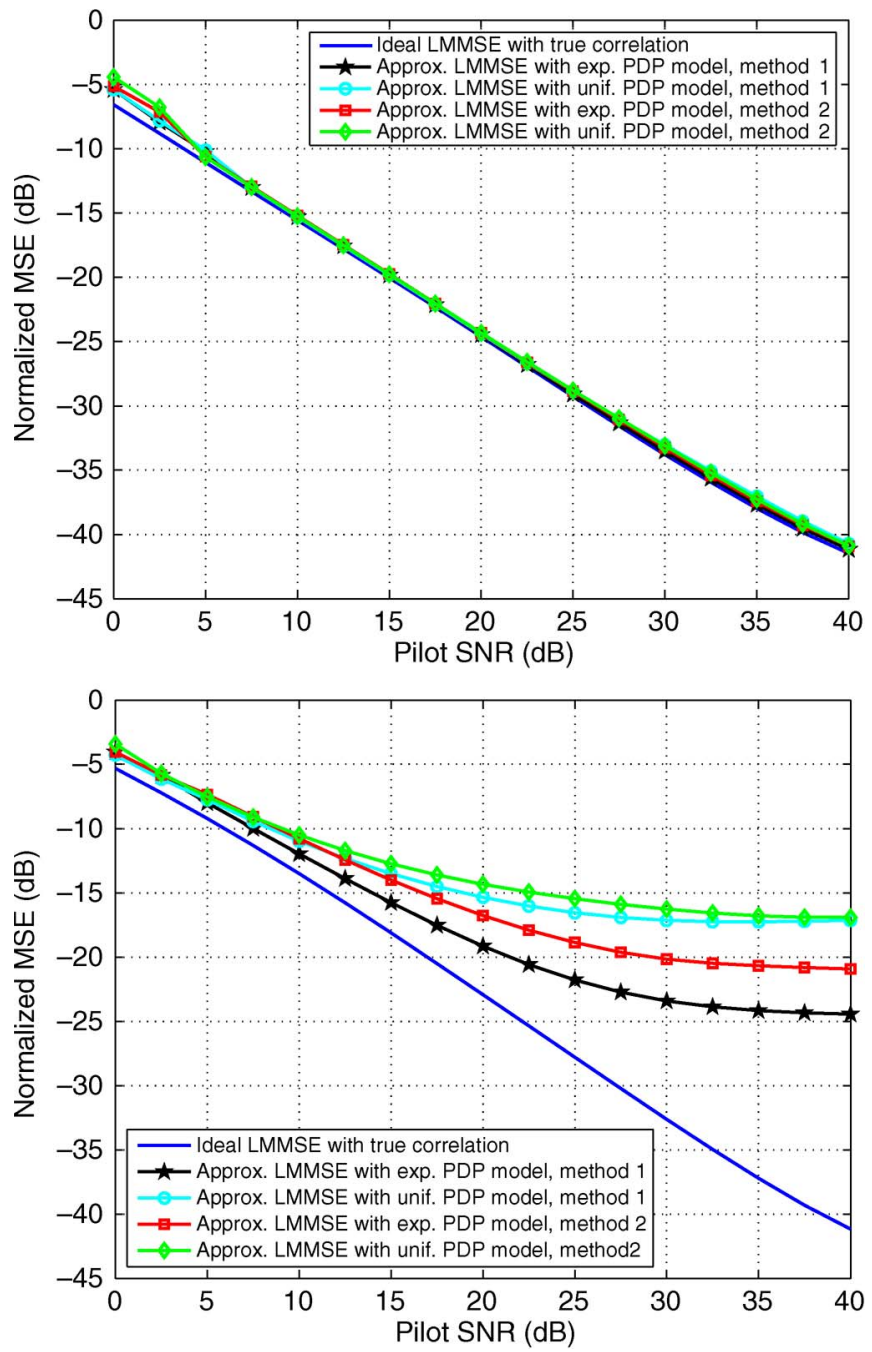

Fig. 6. Channel-estimation performance of different schemes for Mobile WiMAX DL transmission at $100-\mathrm{km} / \mathrm{h}$ mobile speed. (Top) Vehicular A. (Bottom) SUI-5.

ideal LMMSE estimation maintains a slope close to -1 in NMSE throughout the simulated range of SNRs. Overall, the proposed technique performs best with exponential PDP modeling and "bisymbol" reestimation of channel delay parameters.

Now, although the PDPs of the Vehicular A and SUI-5 channels are not exactly exponential, they are nevertheless relatively fast-decreasing functions of delay. This should account for the aforementioned advantage of exponential PDP modeling. To see how the proposed technique may perform in atypical conditions, we create an artificial PDP that consists of three copies of Vehicular A's PDP with "intercluster" delays of 2 and $4 \mu \mathrm{s}$, respectively. The relative power scales of the three "clusters" are $-5,0$, and $-7 \mathrm{~dB}$, respectively. Fig. 7 shows the PDP, and Fig. 8 shows the performance of various channel estimators. While exponential PDP modeling still performs better than uniform modeling, the performance gap is narrower than in SUI-5. Nonetheless, the proposed technique is still applicable.

\section{B. UL Channel Estimation}

As in DL channel estimation, since tiles are pseudorandomly distributed in frequency, we separately perform UL channel

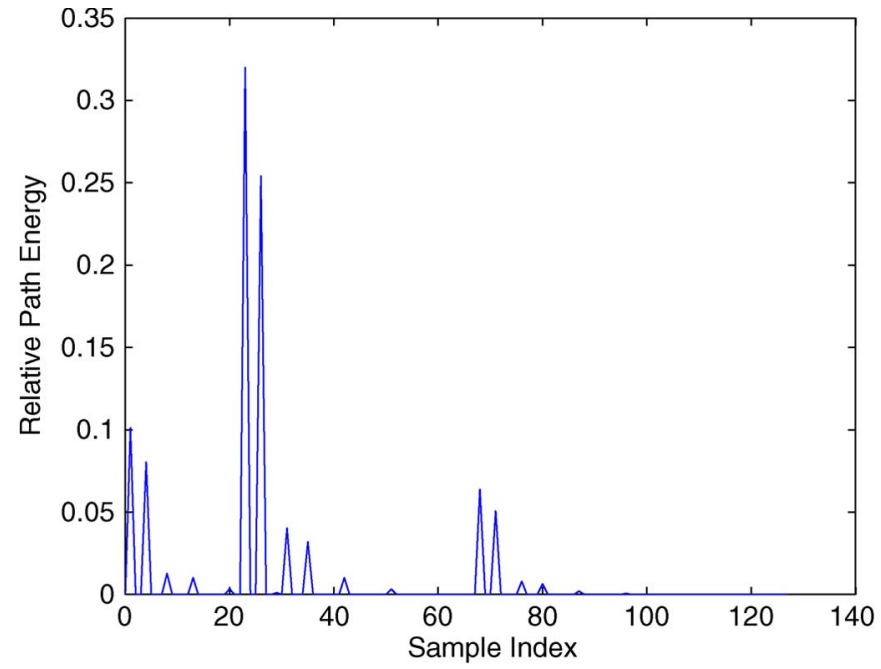

Fig. 7. Artificial PDP created from Vehicular A's PDP.

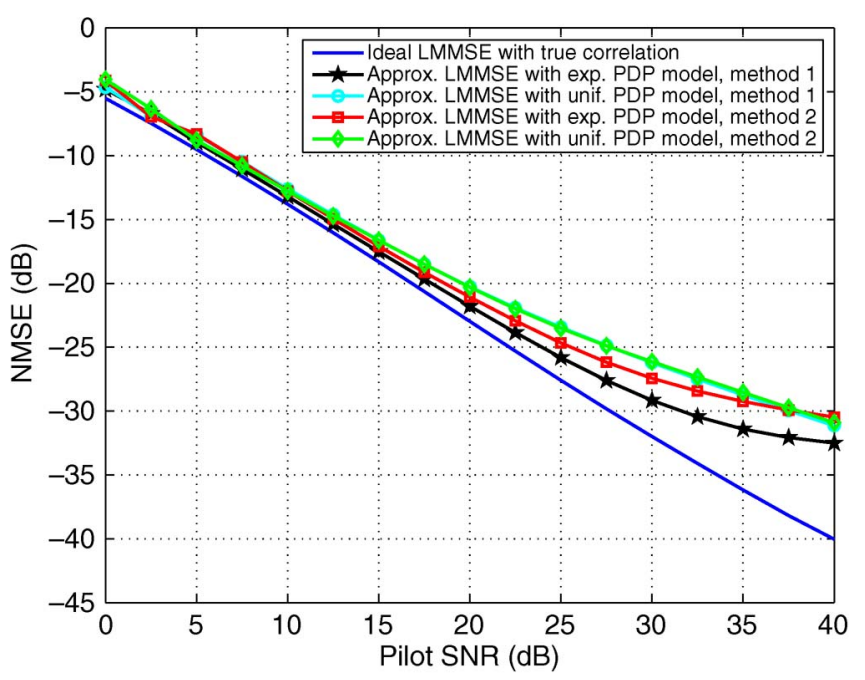

Fig. 8. Channel estimation performance of different schemes for Mobile WiMAX DL transmission at 100-km/h mobile speed in the artificial channel.

estimation for each tile. Since there are at most two pilots in each tile, two-tap Wiener filters are used. The proposed UL channel estimation procedure, over each three symbols wherein tile structures are defined, is given as follows.

1) Estimate the noise variance from the null subcarriers.

2) Do LS channel estimation at the pilot subcarriers in the first and third OFDM symbols of a user.

3) Estimate the mean delay and the RMS delay spread for each user using the aforementioned pilot subcarrier channel estimates and obtain the two-tap Wiener channel estimators for the data subcarriers. (There are a total of two such Wiener filters.)

4) Perform Wiener filtering in the first and third symbols to estimate the data subcarrier responses there.

5) Linearly interpolate in time to obtain channel estimates for the second symbol.

We simulate a system of the same parameters as in the last section, with two subchannels (containing 12 tiles) allocated to a certain user. For both channels (Vehicular A and SUI-5), we allow an initial delay of nine samples (roughly $0.8 \mu \mathrm{s}$ ). 

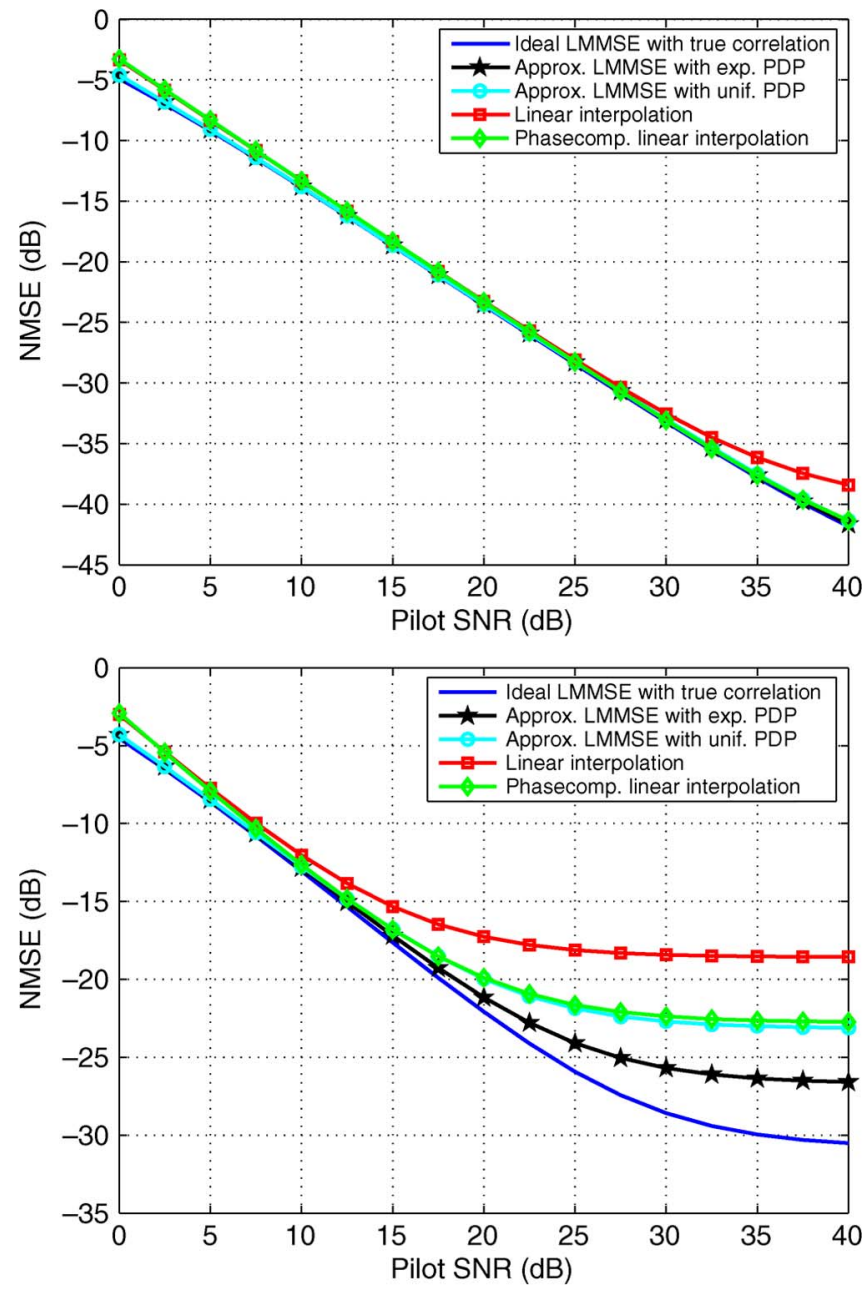

Fig. 9. Channel estimation performance of different schemes for Mobile WiMAX UL transmission at $100-\mathrm{km} / \mathrm{h}$ mobile speed. (Top) Vehicular A. (Bottom) SUI-5.

Fig. 9 illustrates some simulation results, where the proposed technique is compared with three other methods: ideal LMMSE channel estimation based on the true channel correlation (the benchmarker), conventional linear interpolation, and phase-compensated linear interpolation of Hsieh and Wei [21]. The benchmarker aside, in both channels, the proposed technique with exponential PDP modeling performs the best. Approximate LMMSE with uniform PDP modeling and phasecompensated linear interpolation are approximately on par. Furthermore, as may be expected, conventional linear interpolation trails behind all other methods. The performance of all methods degrades as the channel delay spread increases.

\section{CONCLUSION}

We have proposed an LMMSE channel-estimation technique for multicarrier transmission with interspersed pilots among data subcarriers. The technique provides an instrumentable approximation to ideal LMMSE channel estimation. Operating on frequency-domain quantities, it is not only suitable for systems with distributed pilots but also applicable to multiaccess communication. Furthermore, it can comfortably handle the situation where there are only a low number of pilots. Two issues left for further work are the intelligent selection of a proper
PDP model (e.g., between exponential and uniform) under given transmission conditions and methods for improving the channel-estimation accuracy in channels with large delay spreads.

\section{APPENDIX}

We derive the estimation accuracy of $\hat{\tau}_{\mu}$ and $\hat{\tau}_{\text {rms }}$ in this Appendix. From the derivation leading to (19) and (20), we see that estimation errors may arise from three factors: 1) approximating frequency differentiation by finite differencing; 2) approximating frequency averaging by averaging over pilot subcarriers [compare (14) with (12)]; and 3) AWGN [from the use of the estimated $R_{0}$ and $R_{1}$ in (18)]. Among these, factor 2 amounts to using a frequency-sampled signal to compute the original signal's statistics. As noted under (16), by sampling theory, no error will result of it if $L-1<N / F_{s}$. Since OFDM systems are usually designed to have CPs longer than channel impulse responses, the condition is usually satisfied when the pilot subcarrier spacing $F_{s} \leq N /$ (CP length). We assume that this is the case and disregard factor 2 in the analysis.

For convenience, let $\bar{\tau}_{\mu}$ and $\bar{\tau}_{\text {rms }}$ denote the AWGN-free estimates of $\tau_{\mu}$ and $\tau_{\mathrm{rms}}$, respectively. That is, they are obtained with $E\left(R_{i}\right)$ in place of $\operatorname{Av}\left(\hat{R}_{i}\right), i=0,1$, in (19) and (20). Note, however, that $E\left(R_{i}\right)=\operatorname{Av}\left(R_{i}\right), i=0,1$ by the discussion immediately following (5).

\section{A. Estimation Accuracy of Mean Delay}

For the estimation error in $\hat{\tau}_{\mu}$, we have

$$
\Delta \tau_{\mu} \triangleq \hat{\tau}_{\mu}-\tau_{\mu}=\underbrace{\left(\bar{\tau}_{\mu}-\tau_{\mu}\right)}_{\triangleq \delta_{1}}+\underbrace{\left(\hat{\tau}_{\mu}-\bar{\tau}_{\mu}\right)}_{\triangleq e_{1}}
$$

where $\delta_{1}$ is due to finite differencing (factor 1 ), and $e_{1}$ is due to AWGN (factor 3). Therefore, $\delta_{1}$ is a constant, whereas $e_{1}$ is a random variable.

Consider $\delta_{1}$ first

$$
\delta_{1}=-\frac{N}{2 \pi F_{s}} \angle E\left(R_{1}\right)-\tau_{\mu} .
$$

While this expression suffices, because it contains both timedomain and frequency-domain channel parameters, below, we derive an expression in terms of solely time-domain parameters. For this, note that, by Parseval's theorem

$$
E\left(R_{1}\right)=\sum_{l} E\left(\left|\alpha_{l}\right|^{2}\right) e^{-j 2 \pi l F_{s} / N} .
$$

Retaining up to the third-order term in the power series expansion of $e^{j 2 \pi l F_{s} / N}$, we obtain

$$
\begin{aligned}
\angle E\left(R_{1}\right) \approx & \arctan \frac{\frac{-2 \pi F_{s}}{N} \sum_{l} E\left(\left|\alpha_{l}\right|^{2}\right) l+\frac{8 \pi^{3} F_{s}^{3}}{6 N^{3}} \sum_{l} E\left(\left|\alpha_{l}\right|^{2}\right) l^{3}}{\sum_{l} E\left(\left|\alpha_{l}\right|^{2}\right)-\frac{4 \pi^{2} F_{s}^{2}}{2 N^{2}} \sum_{l} E\left(\left|\alpha_{l}\right|^{2}\right) l^{2}} \\
\approx & -\frac{2 \pi F_{s}}{N} \tau_{\mu}+\frac{1}{3}\left(\frac{2 \pi F_{s}}{N} \tau_{\mu}\right)^{3}-\frac{2 \pi F_{s}}{N} \tau_{\mu} \\
& \cdot \frac{4 \pi^{2} F_{s}^{2}}{2 N^{2}} \frac{\sum_{l} E\left(\left|\alpha_{l}\right|^{2}\right) l^{2}}{\sum_{l} E\left(\left|\alpha_{l}\right|^{2}\right)}+\frac{2 \pi F_{s}}{N} \tau_{\mu} \\
& \cdot \frac{4 \pi^{2} F_{s}^{2}}{6 N^{2}} \frac{\sum_{l} E\left(\left|\alpha_{l}\right|^{2}\right) l^{3}}{\sum_{l} E\left(\left|\alpha_{l}\right|^{2}\right) l}
\end{aligned}
$$




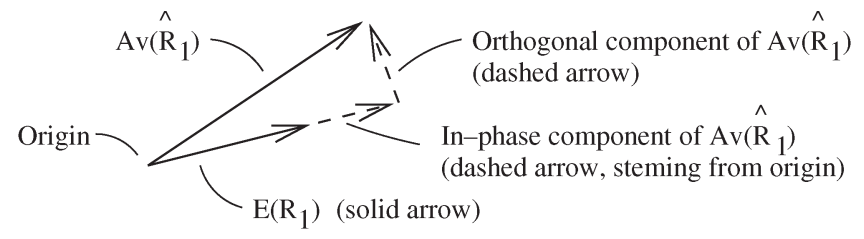

Fig. 10. Computation of angular difference between $E\left(R_{1}\right)$ and $\operatorname{Av}(\hat{R} 1)$.

where the last equality is obtained by approximating the arctan function with the first two terms of its power series expansion. Therefore

$\delta_{1}=\frac{4 \pi^{2} F_{s}^{2}}{N^{2}}\left[\frac{\tau_{\mu}^{3}}{3}-\frac{\tau_{\mu}}{2} \frac{\sum_{l} E\left(\left|\alpha_{l}\right|^{2}\right) l^{2}}{\sum_{l} E\left(\left|\alpha_{l}\right|^{2}\right)}+\frac{1}{6} \frac{\sum_{l} E\left(\left|\alpha_{l}\right|^{2}\right) l^{3}}{\sum_{l} E\left(\left|\alpha_{l}\right|^{2}\right)}\right]$.

Now, consider $e_{1}$. We have

$$
e_{1}=-\frac{N}{2 \pi F_{s}}\left[\angle \operatorname{Av}\left(\hat{R}_{1}\right)-\angle E\left(R_{1}\right)\right] .
$$

Let $W(f)$ denote the AWGN in the estimated channel response $\hat{H}(f)$ at pilot subcarrier $f$. Then

$$
\begin{aligned}
\operatorname{Av}\left(\hat{R}_{1}\right)= & \operatorname{Av}\left\{\left\langle\left[H\left(\left(f+F_{s}\right) \% N\right)+W\left(\left(f+F_{s}\right) \% N\right)\right]\right.\right. \\
& \left.\left.\cdot[H(f)+W(f)]^{*}\right\rangle_{p}\right\} \\
= & E\left(R_{1}\right)+\eta_{1}+\varepsilon_{1}
\end{aligned}
$$

where we have used the previously noted fact that $\operatorname{Av}\left(R_{1}\right)=$ $E\left(R_{1}\right)$ and have let

$$
\begin{aligned}
\eta_{1}=\operatorname{Av}\left\langle H\left(\left(f+F_{s}\right) \% N\right) W^{*}(f)\right. \\
\left.+W\left(\left(f+F_{s}\right) \% N\right) H^{*}(f)\right\rangle_{p} \\
\varepsilon_{1}=\operatorname{Av}\left\langle W\left(\left(f+F_{s}\right) \% N\right) W^{*}(f)\right\rangle_{p} .
\end{aligned}
$$

To find the angular difference between $\operatorname{Av}\left(\hat{R}_{1}\right)$ and $E\left(R_{1}\right)$, we may treat them as vectors in the complex plane and decompose $\operatorname{Av}\left(\hat{R}_{1}\right)$ into the sum of an in-phase and an orthogonal component to $E\left(R_{1}\right)$. This is illustrated in Fig. 10. Let $V_{i}$ and $V_{o}$ denote the values of the in-phase and orthogonal components, respectively. We have

$$
\begin{aligned}
V_{i} & =\Re\left\{\operatorname{Av}\left(\hat{R}_{1}\right) \frac{E\left(R_{1}^{*}\right)}{\left|E\left(R_{1}\right)\right|}\right\} \\
& =\left|E\left(R_{1}\right)\right|+\Re\left\{\frac{\left(\eta_{1}+\varepsilon_{1}\right) E\left(R_{1}^{*}\right)}{\left|E\left(R_{1}\right)\right|}\right\} \\
V_{o} & =\Im\left\{\operatorname{Av}\left(\hat{R}_{1}\right) \frac{E\left(R_{1}^{*}\right)}{\left|E\left(R_{1}\right)\right|}\right\} \\
& =\Im\left\{\frac{\left(\eta_{1}+\varepsilon_{1}\right) E\left(R_{1}^{*}\right)}{\left|E\left(R_{1}\right)\right|}\right\} .
\end{aligned}
$$

Hence

$$
\begin{aligned}
\angle \operatorname{Av}\left(\hat{R}_{1}\right)-\angle E\left(R_{1}\right) & =\arctan \frac{V_{o}}{V_{i}} \\
& \approx \frac{1}{\left|E\left(R_{1}\right)\right|^{2}} \Im\left\{\left(\eta_{1}+\varepsilon_{1}\right) E\left(R_{1}^{*}\right)\right\}
\end{aligned}
$$

where the right-hand side is a first-order approximation in high SNRs. As a result, by the white Gaussianness of $W(f)$, we can show via routine algebra that $E\left(e_{1}\right)=0$ and that

$\sigma_{e_{1}}^{2} \triangleq E\left(\left|e_{1}\right|^{2}\right)=\frac{N^{2}}{8 \pi^{2} F_{s}^{2}\left|E\left(R_{1}\right)\right|^{2}}$

$\times\left[E\left(\left|\eta_{1}\right|^{2}\right)+E\left(\left|\varepsilon_{1}\right|^{2}\right)-\Re\left\{E\left(\eta_{1}^{2}\right) e^{-j 2 \angle E\left(R_{1}\right)}\right\}\right]$

which eventually leads to

$\sigma_{e_{1}}^{2}=\frac{N \sigma_{n}^{2}}{8 \pi^{2} F_{s} K\left|E\left(R_{1}\right)\right|^{2}}\left[\sigma_{n}^{2}+2 E\left(R_{0}\right)-2 \Re\left\{E\left(R_{2}\right) e^{-j 2 \angle E\left(R_{1}\right)}\right\}\right]$.

Moreover, in high SNRs where $\varepsilon_{1}$ is negligible, $e_{1}$ is Gaussian.

\section{B. Estimation Accuracy of RMS Delay Spread}

In the case of $\hat{\tau}_{\text {rms }}$, the error in $\hat{\tau}_{\text {rms }}^{2}$ appears easier to analyze than that in $\hat{\tau}_{\text {rms }}$. We have

$$
\begin{aligned}
\Delta \tau_{\mathrm{rms}}^{2} & \triangleq \hat{\tau}_{\mathrm{rms}}^{2}-\tau_{\mathrm{rms}}^{2} \\
& =\underbrace{\left(\bar{\tau}_{\mathrm{rms}}^{2}-\tau_{\mathrm{rms}}^{2}\right)}_{\triangleq \delta_{2}}+\underbrace{\left(\hat{\tau}_{\mathrm{rms}}^{2}-\bar{\tau}_{\mathrm{rms}}^{2}\right)}_{\triangleq e_{2}}
\end{aligned}
$$

where $\delta_{2}$ is due to finite differencing (factor 1 given earlier), and $e_{2}$ is due to AWGN (factor 3 given earlier). Again, the former is a constant, while the latter is a random variable.

For $\delta_{2}$, we have

$$
\delta_{2}=\frac{2 N^{2}}{4 \pi^{2} F_{s}^{2}}\left[1-\frac{\left|E\left(R_{1}\right)\right|}{E\left(R_{0}\right)}\right]-\tau_{\mathrm{rms}}^{2} .
$$

Again, this expression mixes time-domain and frequencydomain channel parameters. Therefore, we derive an expression in terms of only time-domain parameters. To start, note that

$$
\begin{aligned}
\left|E\left(R_{1}\right)\right| & =E\left(R_{1}\right) e^{-j \angle E\left(R_{1}\right)} \\
& =\sum_{l} E\left(\left|\alpha_{l}\right|^{2}\right) e^{j 2 \pi\left(\bar{\tau}_{\mu}-l\right) F_{s} / N} .
\end{aligned}
$$

Retaining up to the fourth-order terms of the series expansions of various transcendental functions involved (details omitted), we finally get

$$
\begin{aligned}
\left|E\left(R_{1}\right)\right| \approx E\left(R_{0}\right) & {\left[1-\frac{4 \pi^{2} F_{s}^{2}}{2 N^{2}} \tau_{\mathrm{rms}}^{2}\right.} \\
& \left.+\frac{16 \pi^{4} F_{s}^{4}}{24 N^{4} E\left(R_{0}\right)} \sum_{l} E\left(\left|\alpha_{l}\right|^{2}\right)\left(l-\tau_{\mu}\right)^{4}\right] .
\end{aligned}
$$

Then, substituting this last result into (39) gives

$$
\delta_{2}=-\frac{\pi^{2} F_{s}^{2}}{3 N^{2} E\left(R_{0}\right)} \sum_{l} E\left(\left|\alpha_{l}\right|^{2}\right)\left(l-\tau_{\mu}\right)^{4} .
$$


For $e_{2}$,we get

$$
e_{2}=\frac{2 N^{2}}{4 \pi^{2} F_{s}^{2}}\left[\frac{\left|E\left(R_{1}\right)\right|}{E\left(R_{0}\right)}-\frac{\left|\operatorname{Av}\left(\hat{R}_{1}\right)\right|}{\operatorname{Av}\left(\hat{R}_{0}\right)}\right] .
$$

To find its statistics, we express $\operatorname{Av}\left(\hat{R}_{0}\right)$ and $\left|\operatorname{Av}\left(\hat{R}_{1}\right)\right|$ as perturbations of $E\left(R_{0}\right)$ and $\left|E\left(R_{1}\right)\right|$. For $\operatorname{Av}\left(\hat{R}_{0}\right)$, assuming that $\hat{\sigma}_{n}^{2}$ is an accurate estimate of $\sigma_{n}^{2}$, i.e., $\hat{\sigma}_{n}^{2}=\sigma_{n}^{2}$, we get

$$
\begin{aligned}
\operatorname{Av}\left(\hat{R}_{0}\right) & =\operatorname{Av}\left\langle|H(f)+W(f)|^{2}\right\rangle_{p}-\sigma_{n}^{2} \\
& =E\left(R_{0}\right)+\eta_{0}+\varepsilon_{0}
\end{aligned}
$$

with

$$
\begin{aligned}
& \eta_{0}=\operatorname{Av}\left\langle H(f) W^{*}(f)+W(f) H^{*}(f)\right\rangle_{p} \\
& \varepsilon_{0}=\operatorname{Av}\left\langle|W(f)|^{2}\right\rangle_{p}-\sigma_{n}^{2} .
\end{aligned}
$$

For $\left|\operatorname{Av}\left(\hat{R}_{1}\right)\right|$, from the earlier discussion concerning $e_{1}$, we have $\left|\operatorname{Av}\left(\hat{R}_{1}\right)\right|=\sqrt{V_{i}^{2}+V_{o}^{2}}$. Thus, with high SNRs, $\left|\operatorname{Av}\left(\hat{R}_{1}\right)\right| \approx V_{i}$. Combined with (44)

$$
\frac{\left|\operatorname{Av}\left(\hat{R}_{1}\right)\right|}{\operatorname{Av}\left(\hat{R}_{0}\right)} \approx \frac{\left|E\left(R_{1}\right)\right|}{E\left(R_{0}\right)}\left[1+\frac{\Re\left\{\left(\eta_{1}+\varepsilon_{1}\right) E\left(R_{1}^{*}\right)\right\}}{\left|E\left(R_{1}\right)\right|^{2}}-\frac{\eta_{0}+\varepsilon_{0}}{E\left(R_{0}\right)}\right] .
$$

Substituting into (43) yields

$$
e_{2} \approx \frac{N^{2}}{2 \pi^{2} F_{s}^{2}} \frac{\left|E\left(R_{1}\right)\right|}{E\left(R_{0}\right)}\left[\frac{\eta_{0}+\varepsilon_{0}}{E\left(R_{0}\right)}-\frac{\Re\left\{\left(\eta_{1}+\varepsilon_{1}\right) E\left(R_{1}^{*}\right)\right\}}{\left|E\left(R_{1}\right)\right|^{2}}\right] .
$$

Then, it can be shown via routine algebra that $E\left(e_{2}\right)=0$ and that

$$
\begin{aligned}
\sigma_{e_{2}}^{2} \triangleq & E\left(\left|e_{2}\right|^{2}\right)=\frac{N^{3} \sigma_{n}^{2}}{8 \pi^{4} F_{s}^{3} K E\left(R_{0}\right)^{4}} \\
\times & \left\{\left[E\left(R_{0}\right)^{2}+2\left|E\left(R_{1}\right)\right|^{2}\right] \sigma_{n}^{2}+2 E\left(R_{0}\right)^{3}\right. \\
& -4 E\left(R_{0}\right)\left|E\left(R_{1}\right)\right|^{2}+2 E\left(R_{0}\right)^{2} \\
& \left.. \Re\left\{E\left(R_{2}\right) e^{-j 2 \angle E\left(R_{1}\right)}\right\}\right\} .
\end{aligned}
$$

With high SNRs where $\varepsilon_{0}$ and $\varepsilon_{1}$ are negligible, $e_{2}$ is Gaussian.

\section{REFERENCES}

[1] P. Hoeher, S. Kaiser, and P. Robertson, "Two-dimensional pilot-symbolaided channel estimation by Wiener filtering," in Proc. IEEE Int. Conf. Acoust., Speech, Signal Process., 1997, vol. 3, pp. 1845-1848.

[2] M. Morelli and U. Mengali, "A comparison of pilot-aided channel estimation methods for OFDM systems," IEEE Trans. Signal Process., vol. 49, no. 12 , pp. 3065-3073, Jan. 1998

[3] O. Edfors, M. Sandell, J.-J. van de Beek, S. K. Wilson, and P. O. Börjesson, "OFDM channel estimation by singular value decomposition," IEEE Trans. Commun., vol. 46, no. 7, pp. 931-939, Jul. 1998.

[4] Y. Li, "Pilot-symbol-aided channel estimation for OFDM in wireless systems," IEEE Trans. Veh. Technol., vol. 49, no. 4, pp. 1207-1215, Jul. 2000.

[5] H. Stark and J. W. Woods, Probability and Random Processes, 3rd ed. Upper Saddle River, NJ: Prentice-Hall, 2002.

[6] R. van Nee and R. Prasad, OFDM for Wireless Multimedia Communications. Boston, MA: Artech House, 2000.
[7] L. Wilhelmsson, B. Bernhardsson, and L. Andersson, "Channel estimation by adaptive interpolation,” U.S. Patent 7433 433, Oct. 7, 2008.

[8] Y.-J. Bang, K.-Y. Sohn, and Y.-O. Park, "Apparatus and method of estimating channel based on channel delay spread in mobile communication system," U.S. Patent Pub. No. 2008/0137788, Jun. 12, 2008.

[9] R. Akella and R. Cheng, "Edge MMSE filters," U.S. Patent Pub. No. 2008/0144730, Jun. 19, 2008

[10] K. Witrisal, "On estimating the RMS delay spread from the frequencydomain level crossing rate," IEEE Commun. Lett., vol. 5, no. 7, pp. 287289, Jul. 2001.

[11] K. Witrisal, Y.-H. Kim, and R. Prasad, "A new method to measure parameters of frequency-selective radio channels using power measurements," IEEE Trans. Commun., vol. 49, no. 10, pp. 1788-1800, Oct. 2001.

[12] C. N. R. Athaudage and A. D. S. Jayalath, "Delay-spread estimation using cyclic-prefix in wireless OFDM systems," Proc. Inst. Elect. Eng.-Commun., vol. 151, no. 6, pp. 559-566, Dec. 2004.

[13] T. Yücek and H. Arslan, "Time dispersion and delay spread estimation for adaptive OFDM systems," IEEE Trans. Veh. Technol., vol. 57, no. 3, pp. 1715-1722, May 2008

[14] IEEE Standard for Local and Metropolitan Area Networks-Part 16 Air Interface for Fixed and Mobile Broadband Wireless Access Systems-Amendment 2: Physical and Medium Access Control Layers for Combined Fixed and Mobile Operation in Licensed Bands and Corrigendum 1, IEEE Stds. 802.16e-2005 and 802.16-2004/Cor1-2005, Feb. 28, 2006.

[15] WiMAX Forum, Mobile WiMAX_-Part I: A Technical Overview and Performance Evaluation, Aug. 2006.

[16] A. V. Oppenheim, R. W. Schafer, and J. R. Buck, Discrete-Time Signal Processing, 2nd ed. Upper Saddle River, NJ: Prentice-Hall, 1999.

[17] T. B. Sørensen, P. E. Mogensen, and F. Frederiksen, "Extension of the ITU channel models for wideband (OFDM) systems," in Proc. IEEE Veh. Technol. Conf., Sep. 2005, pp. 392-396.

[18] Channel Models for Fixed Wireless Applications, IEEE 802.16.3c-01/29r4, Jul. 17, 2001.

[19] Y. Mostofi and D. C. Cox, "ICI mitigation for pilot-aided OFDM mobile systems," IEEE Trans. Wireless Commun., vol. 4, no. 2, pp. 765-774, Mar. 2005.

[20] K.-C. Hung and D. W. Lin, "Optimal delay estimation for phase-rotated linearly interpolative channel estimation in OFDM and OFDMA systems," IEEE Signal Process. Lett., vol. 15, pp. 349-352, 2008.

[21] M.-H. Hsieh and C.-H. Wei, "Channel estimation for OFDM systems based on comb-type pilot arrangement in frequency selective fading channels," IEEE Trans. Consum. Electron., vol. 44, no. 1, pp. 217-225, Feb. 1998.

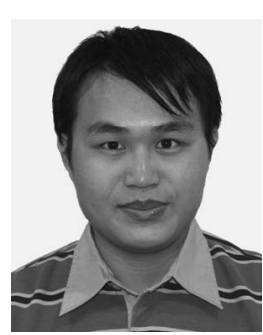

Kun-Chien Hung received the B.S. and Ph.D. degrees in electronics engineering from the National Chiao Tung University, Hsinchu, Taiwan, in 2001 and 2008, respectively.

$\mathrm{He}$ is currently a Senior Engineer with MediaTek Inc., Hsinchu. His research interests are in digital signal processing, communication receiver design, and wireless communication.

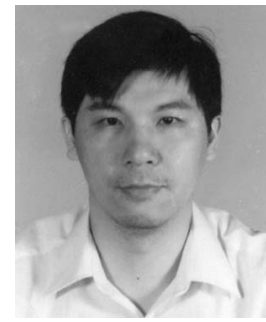

David W. Lin (S'79-M'81-SM'88) received the B.S. degree from the National Chiao Tung University, Hsinchu, Taiwan, in 1975 and the M.S. and Ph.D. degrees in electrical engineering from the University of Southern California, Los Angeles, in 1979 and 1981, respectively.

From 1981 to 1983, he was with Bell Laboratories. From 1984 to 1990 and again from 1993 to 1994 he was with Bellcore. Since 1990, he has been a Professor with the Department of Electronics Engineering, National Chiao Tung University, except for a leave in 1993-1994. He has conducted research in digital adaptive filtering and telephone echo cancellation, digital subscriber line and coaxial network transmission, speech and video coding, and wireless communication. His research interests include various topics in signal processing and communication engineering. 